\section{ORIGINAL RESEARCH}

\author{
R. Yasuda \\ K. Royalty \\ K. Pulfer \\ D. Consigny
}

C.M. Strother

\title{
C-Arm CT Measurement of Cerebral Blood Volume Using Intra-Arterial Injection of Contrast Medium: An Experimental Study in Canines
}

\begin{abstract}
BACKGROUND AND PURPOSE: Measurement of perfusion parameters is typically done using an intravenous injection of contrast medium. This purpose of this study was to evaluate the feasibility of measuring regional and global CBV using C-arm CT with IA injections of contrast medium.
\end{abstract}

\begin{abstract}
MATERIALS AND METHODS: Twelve canines were studied. CBV measurement was performed using standard PCT, and then using C-arm CT with IV and IA contrast. Values obtained using C-arm CT were compared with those using PCT.
\end{abstract}

\begin{abstract}
RESULTS: C-arm CT CBV maps using IA injections required less contrast than ones with IV injections. PCT and $\mathrm{C}$-arm CT using IV and AA injections provided comparable maps. In controls, C-arm CT with a CCA or VA injection provided comparable maps to PCT. In animals with a stroke, a CCA or VA injection did not provide maps comparable to ones made with PCT. IV and AA C-arm CT showed excellent quantitative agreement with PCT, while CCA and VA C-arm CT studies did not.
\end{abstract}

\begin{abstract}
CoNCLUSIONS: Measurement of global CBV using C-arm CT in conjunction with either an IV or an AA injection was feasible in controls and dogs with a stroke. Measurement of regional CBV with $\mathrm{C}$-arm $\mathrm{CT}$ using either CCA or VA injection, in normal canines, provided CBV maps qualitatively comparable with those obtained with PCT; the absolute CBV values from these maps were in poor agreement with PCT measurements. Valid measurement of CBV using $\mathrm{C}$-arm CT requires all tissue in a target region to be fully and equally opacified during any acquisition. Using CCA or VA injections, it was impossible to document if and when this had been achieved. CBV measurements using these routes of injection were therefore not reliable.
\end{abstract}

ABBREVIATIONS: $\mathrm{AA}=$ aortic arch; $\mathrm{CCA}=$ common carotid artery; $\mathrm{GEE}=$ generalized estimating equation; $\mid \mathrm{A}=$ intra-arterial; $\mathrm{PCT}=$ perfusion $\mathrm{CT}$; $\mathrm{VA}=$ vertebral artery

$\mathbf{S}_{\text {iisol }}^{\text {o }}$ olid evidence regarding the evolution of ischemic brain tissue and the benefits of prompt intervention in appropriate patients provides the motivation for seeking techniques that would increase the speed and efficiency of diagnosis and initiation of treatment in patients with an acute ischemic stroke. ${ }^{1-3}$ A key parameter in trying to identify those patients who have ischemic, but potentially viable, brain parenchyma (who thus might benefit from revascularization) from ones who have ischemic brain tissue that is not salvageable is CBV. ${ }^{4,5}$ Animal and human studies have recently demonstrated the feasibility and possible advantage of measuring $\mathrm{CBV}$ in the angiographic suite using C-arm CT. ${ }^{6-9}$ As emphasized by Harris and colleagues, ${ }^{10}$ both the rate and the amount of ischemic core growing into a region of penumbra varies greatly in each patient. Thus, as is the case for perfusion parameters measured with other techniques, the ability to obtain serial C-arm CT measurements of CBV before and then during an intervention could add value in determining the effectiveness of revascularization and also in determining the time

Received December 6, 2011; accepted after revision January 23, 2012.

From the Department of Neurosurgery, Mie University Graduate School of Medicine (R.Y.), Tsu, Mie, Japan; Siemens Medical Solutions (K.R.), Hoffman Estates, Illinois; Department of Radiology, University of Wisconsin School of Medicine and Public Health (K.P., D.C., C.M.S.), Madison, Wisconsin.

Please address correspondence to Charles M. Strother, MD, Department of Radiology, University of Wisconsin School of Medicine and Public Health, 600 North Highland Ave, Madison, WI 53792; e-mail: cstrother@uwhealth.org

http://dx.doi.org/10.3174/ajnr.A3077 when the risk of injury from treatment exceeds further possible benefit. ${ }^{11,12}$

To our knowledge, all previous attempts at measurement of CBV using C-arm CT have been done in association with IV injections of contrast. Because the volume of contrast medium required for perfusion measurement is significant, concerns about contrast toxicity would probably somewhat limit the ability to obtain serial measurements during an intervention. Because the volume and concentration of contrast medium required for an intra-arterial measurement of perfusion are greatly reduced compared with an IV measurement, the use of IA injections would eliminate this concern. The purpose of this study was to test the hypothesis that measurement of global and regional CBV in canines with an acute stroke using selective IA injections of contrast medium would compare well with ones made using either $\mathrm{C}$-arm CT in conjunction with an IV injection of contrast or with standard PCT measurements.

\section{Materials and Methods}

\section{Creation of Ischemia}

Under an institutionally approved protocol, 12 canines were studied. General anesthesia was introduced with a propofol $10-\mathrm{mL} / \mathrm{kg}$ IV injection and maintained using $1 \%-5 \%$ isoflurane and $100 \%$ oxygen. For 8 of the 12 canines, ischemia was created in 1 or both cerebral hemispheres; 4 animals were studied as normal controls and underwent no intervention other than the general anesthesia. To create an infarct a $4 \mathrm{~F}$ catheter (Tempo 4; Cordis, Miami Lakes, Florida) was 


\begin{tabular}{|c|c|c|c|c|c|c|}
\hline \multirow[b]{2}{*}{ Modality } & \multicolumn{3}{|c|}{ Contrast } & \multicolumn{2}{|c|}{ Saline Chase } & \multirow[b]{2}{*}{$\begin{array}{c}\text { Total Injection } \\
\text { Volume (ml) }\end{array}$} \\
\hline & $\begin{array}{c}\text { Concentration } \\
(\%)\end{array}$ & $\begin{array}{l}\text { Volume } \\
\text { (ml) }\end{array}$ & $\begin{array}{l}\text { Injection Rate } \\
(\mathrm{ml} / \mathrm{s})\end{array}$ & $\begin{array}{l}\text { Volume } \\
\text { (ml) }\end{array}$ & $\begin{array}{l}\text { Injection Rate } \\
(\mathrm{ml} / \mathrm{s})\end{array}$ & \\
\hline$\overline{\text { PCT }}$ & 100 & 12 & 1.0 & 10 & 1.5 & 22 \\
\hline C-arm IV & 100 & 25 & 1.0 & 10 & 1.0 & 35 \\
\hline C-arm AA & $15-20$ & $11.25-15$ & 4.0 & & & 75 \\
\hline C-arm CCA & $10-30$ & 4.3-15 & 2.5 & & & $43-50$ \\
\hline C-arm VA & $10-30$ & $3-9.9$ & $1.5-2.5$ & & & $30-33$ \\
\hline
\end{tabular}

placed into either a CCA or a VA. Via this catheter, a microcatheter (Fastracker 18; Target Therapeutics, Fremont, California) was advanced to the origin of the MCA or the ICA and pieces of silk suture and/or collagen particles (Avitene; Bio-Medicine, Houston, Texas) were slowly injected until there was angiographic evidence of stasis of contrast in intracranial arteries.

\section{PCT}

After induction of general anesthesia, the 4 normal canines were transferred immediately to the CT room, where standard PCT measurements of CBV were performed. Four hours after the stroke creation, the 8 canines with a stroke were transferred to the CT room, where standard PCT measurement of CBV was performed using a 64-section volume scanner (GE Healthcare, Milwaukee, Wisconsin). IV injection of iopamidol, $370 \mathrm{mgI} / \mathrm{mL}$ (Isovue 370; Bracco Diagnostics, Princeton, New Jersey) was performed using a dual-syringe power injector (Mark V ProVis; Medrad, Indianola, Pennsylvania). The injection protocol is shown in Table 1. After the injection and after a 5-second prep delay, continuous scanning was initiated using the following parameters: $80 \mathrm{kVp}, 200 \mathrm{~mA}, 1$ second per rotation for 50 seconds.

\section{C-Arm CT}

Immediately after the PCT study, the canines were transferred to the angiographic suite for C-arm CT CBV measurements. (Artis zeego; Siemens, Erlangen, Germany). The same contrast medium as used in PCT was injected either into a peripheral vein, AA, or a selected CCA or VA using a dual syringe power injector (Medtron, Saarbrücken, Germany) as indicated by the injection protocols shown in Table 1. The image acquisition consisted of 2 rotations: an initial rotation (mask run), followed by a second rotation after a contrast injection, and an appropriate $\mathrm{x}$-ray delay (fill run). The $\mathrm{x}$-ray delay was adjusted to acquire images while the target tissue was kept in a steady state of contrast enhancement. To determine the appropriate $\mathrm{x}$-ray delay, a timing run was performed by starting a 2D-DSA acquisition simultaneously with the start of a contrast injection. The time from the start of the injection to the time of good opacification of the superior sagittal sinus was noted, and then an additional 7-10 seconds was added to account for longer transit times resulting from contrast passing through collaterals. This time was then used for the x-ray delay for the animals with strokes. For the normal animals, the x-ray delay was set as the time from the start of an injection to good filling of the superior sagittal sinus. Two hundred forty-eight projections were acquired during the 10 -second rotational acquisition. For the C-arm CT measurement of CBV, 1 or more of the following injections were performed in each animal: IV $(n=9), \mathrm{AA}(n=9)$, CCA $(n=7)$, and VA $(n=10)$.

\section{Postprocessing}

Postprocessing was performed using commercial software (CT perfusion 4, GE Healthcare) for PCT and prototype software (Siemens) for all the C-arm CT studies. The reconstruction algorithm has been previously described in detail. ${ }^{6,7}$ In brief, after reconstruction and subtraction of the mask run and the fill run, an algorithm was applied to further segment out air and bone from the image volume. In the PCT postprocessing, vascular inputs were selected manually. Because of the small size of canine intracranial vessels and the proximity of the superior sagittal sinus to the thick skull base, extracranial vessels were selected for arterial and venous input functions. In the C-arm CT postprocessing, the steady-state arterial and venous input functions were calculated from an automated histogram analysis of the vessel tree. A final scaling was then applied to account for the arterial input, as well as other physiologic values (eg, hematocrit), before a smoothing filter was applied to reduce pixel noise. Vessel components were removed in composing CBV maps for PCT; this was not done for the $\mathrm{C}$-arm studies due to the inherent difference of the postprocessing software.

\section{Section Selection and Region of Interest Placement}

After the postprocessing, 2 sections were chosen from each PCT and C-arm CT study. For the normal canines, sections centered on the anterior horns of the lateral ventricles were selected; as closely as possible, the sections were matched anatomically from animal to animal using commercial image registration (Siemens). For the canines with a stroke, the same technique was used except that the sections were selected such that they were centered as closely as possible on the area of ischemia. ROIs were placed manually by an experienced researcher (K.P.) with more than 10 years of experience in CT imaging. Three circular ROIs $\left(75 \mathrm{~mm}^{2}\right)$ were placed on each hemisphere in an attempt to cover all the tissues and to not include vessels. In addition, the ROIs were set to include the same proportion of gray/white matter as they possess distinct normal range of $\mathrm{CBV}^{13,14}$ as well as threshold of infarct. ${ }^{5,15,16}$ The 3 ROIs on each hemisphere were adjusted to be symmetrical.

\section{Data Analysis}

Both qualitative and quantitative comparisons between CBV measurements acquired using PCT and those acquired using C-arm CT were performed.

For qualitative analysis, 2 expert neuroradiologists (R.B. and B.A.K.) who were not otherwise involved in the study were recruited. After a simple introduction to the project, part of which consisted of being shown typical examples of CBV maps from normal and abnormal canine studies, they were asked to independently review the CBV maps obtained from the study animals. The CBV maps were presented using PowerPoint (Microsoft, Redmond, Washington). The order of the slides was randomized according to a computer-gener- 


\begin{tabular}{lcc}
\hline \multicolumn{3}{l}{ Table 2: Dogs and C-arm injections performed } \\
\hline Dog \# & Stroke Creation & C-Arm Injection Sites \\
\hline 1 & Normal & CCA(L), VA \\
2 & L & CCA(L), VA, IV \\
3 & Normal & CCA(L), VA \\
4 & L, R & AA, IV, VA, CCA(R) \\
5 & R & VA, IV \\
6 & $L$ & $A A, I V, V A, C C A(R)$ \\
7 & L, R & AA, IV, VA \\
8 & L & AA, IV, VA \\
9 & Normal & CCA(L), CCA(R), VA, IV \\
10 & R & AA, IV \\
11 & Normal & CCA(L), CCA(R), VA \\
12 & L, R & AA, IV \\
\hline
\end{tabular}

Note:-PCT was performed in all dogs. $L$ indicates left; $R$, right.

ated scheme. Each slide displayed a pair of maps from 1 of the studies. No information was provided regarding the technique used to generate the map or the route of contrast administration. The observers were asked to independently determine 1) whether the study was normal or if there was evidence of ischemia, and 2) if ischemia was present, on which side (or bilateral) was the abnormality located. Magnification/demagnification of the images was allowed, whereas color adjustment of the images was not allowed.

For quantitative analysis, statistical analysis was performed using R version 2.10.0 (R Development Core Team, http://www.R-project. org) and the "gee" package version 4.13 (Carey, http://CRAN.Rproject.org/package $=$ gee). GEE models were used to test for group differences in CBV between stroke and normal hemispheres. Separate models were fitted to each injection type allowing the comparison (PCT, C-arm IV, and C-arm AA injections). In all the cases, an identity link with Gaussian response, independence working correlation, and robust variance estimates were used; 95\% confidence intervals and Wald test $P$ values were obtained based on the latter. To test for agreement between PCT and the various $\mathrm{C}$-arm injection alternatives, bias and Bland-Altman 95\% limits of agreement between the 2 modalities were obtained and plotted. ${ }^{17,18}$ Both the GEE model and the Bland-Altman limits of agreement took into account the clustering stemming from having repeated measures within an individual canine due to 1) each dog receiving multiple injections, and 2) CBV being measured at 3 ROIs, paired across brain hemispheres at each of 2 sections. A $P$ value (2-sided) less than .05 was used as the criterion for statistical significance.

\section{Results}

Unilateral acute ischemia was created in 6 canines; 2 had bilateral hemispheric ischemia. PCT was performed in all ani-

\begin{tabular}{|c|c|c|c|c|c|}
\hline & РCT & $\begin{array}{l}\text { C-Arm } \\
\text { IV }\end{array}$ & $\begin{array}{c}\text { C-Arm } \\
\text { AA }\end{array}$ & $\begin{array}{l}\text { C-Arm } \\
\text { CCA }\end{array}$ & $\begin{array}{l}\text { C-Arm } \\
\text { VA }\end{array}$ \\
\hline \multicolumn{6}{|l|}{ Observer 1} \\
\hline $\begin{array}{l}\text { True-positive } \\
\text { False-positive }\end{array}$ & 8 & 7 & 6 & & 2 \\
\hline False-negative & & 1 & & 2 & 3 \\
\hline True-negative & 4 & 1 & & 7 & 4 \\
\hline \multicolumn{6}{|l|}{ Observer 2} \\
\hline $\begin{array}{l}\text { True-positive } \\
\text { False-positive }\end{array}$ & 8 & 8 & 5 & & 3 \\
\hline False-negative & & & 1 & 2 & 2 \\
\hline True-negative & 4 & 1 & & 7 & 4 \\
\hline
\end{tabular}

Table 4: Mean CBV values in each modality

\begin{tabular}{lccc}
\hline Modality & Normal Hemisphere & Stroke Hemisphere & $P$ value $^{\text {a }}$ \\
\hline PCT & $2.38 \pm 0.54(n=14)$ & $1.01 \pm 0.85(n=10)$ & $<0.001$ \\
C-arm IV & $2.33 \pm 0.67(n=8)$ & $1.00 \pm 0.76(n=10)$ & $<0.001$ \\
C-arm AA & $2.47 \pm 0.76(n=4)$ & $1.02 \pm 0.93(n=8)$ & $<0.001$ \\
C-arm CCA & $2.40 \pm 0.73(n=7)$ & - & - \\
C-arm VA & $2.23 \pm 1.21(n=10)$ & - & - \\
\hline
\end{tabular}

${ }^{a}$ General estimating equation model. The numbers in the parentheses represent hemispheres studied.

mals. One or more C-arm study was also performed in each animal. Table 2 shows the location of the ischemic areas along with the injection sites that were used for C-arm CT acquisitions in each animal (Table 2).

For each of the various $\mathrm{C}$-arm acquisitions, the amount of contrast medium used is shown in Table 1. Measurements made using any of the IA injection sites resulted in a marked reduction in contrast dose for a single acquisition compared with that required for a measurement made with an IV injection. The most commonly used injection parameters for acquisitions made with the VA or CCA sites also resulted in considerable reductions in contrast dose compared with the AA injections.

The 2 expert observers correctly graded all PCT studies of both the normal controls and the animals with acute ischemia. Of the 9 C-arm CT studies completed with an IV injection in the animals with acute ischemia, only 1 was graded incorrectly (observer 1); likewise, of the $6 \mathrm{C}$-arm CT studies completed with an AA injection in the animals with acute ischemia, only 1 was graded incorrectly (observer 2). Both observers graded all of the C-arm CT studies of the normal animals completed with a CCA or a VA injection correctly. In the animals with acute ischemia, 3 studies were completed in conjunction with a CCA injection and 6 studies were completed in conjunction with a VA injection. In 2 with a CCA injection and in 2 of the 6 with a VA injection, no contrast was observed in the hemisphere containing acute ischemia, that is, there was no collateral filling. Both of these CCA studies were graded incorrectly; both of the VA studies were also graded incorrectly. The other CBV maps acquired using a VA injection were qualitatively comparable with those obtained using PCT. In every instance where there was an error in grading of the studies completed in conjunction with a CCA or a VA study, angiography confirmed that there was insufficient collateral supply to the relevant hemisphere to provide adequate tissue opacification from the injected artery (Table 3 ). In the correctly graded studies completed with a CCA or VA injection, angiography confirmed good tissue opacification of the relevant hemisphere by way of collateral supply. Because of this inconsistency in achieving significant and stable tissue opacification (and, thus, valid CBV maps), we did not perform statistical analysis of the region-of-interest values in the hemisphere with a stroke in the studies completed in conjunction with a CCA or a VA injection.

The mean CBV values extracted from each technique are summarized in Table 4. In all of the PCT, C-arm IV, and AA studies, CBV values in the normal hemispheres were significantly higher than were those in the hemisphere with acute ischemia (PCT: $2.38 \pm 0.54$ versus $1.01 \pm 0.85, P<.001$; C-arm IV: $2.33 \pm 0.67$ versus $1.00 \pm 0.76, P<.001$; C-arm 


\begin{tabular}{lccc}
\hline Table 5: Bland-Altman analysis & & \multicolumn{2}{c}{$\begin{array}{c}\text { 95\% Limits of } \\
\text { Agreement }\end{array}$} \\
\cline { 3 - 4 } & & Lower & Upper \\
\hline PCT vs C-arm IV & $-0.031(0.447)$ & -1.218 & 1.155 \\
PCT vs C-arm AA & $-0.092(0.605)$ & -1.630 & 1.446 \\
PCT vs C-arm CCA & $0.048(0.912)$ & -2.055 & 2.153 \\
PCT vs C-arm VA & $0.180(1.463)$ & -3.105 & 3.465 \\
\hline
\end{tabular}

AA: $2.47 \pm 0.76$ versus $1.02 \pm 0.93, P<.001$ ) (Table 4 ). In quantitative assessments, biases and 95\% limits of agreement in Bland-Altman analyses were tabulated (Table 5) and scatterplots are displayed (Fig 1). The limits of agreement were narrowest for C-arm IV injections, closely followed by C-arm AA injections (standard deviations of $\mathrm{CBV}$ difference were 0.447 and $0.605 \mathrm{~mL} / 100 \mathrm{~g}$, respectively). Biases in these $2 \mathrm{mo}-$ dalities were also minimal $(-0.031$ and $-0.092 \mathrm{~mL} / 100 \mathrm{~g}$, respectively) and most of the plots were within the limits of agreement. On the other hand, agreement of PCT with C-arm $\mathrm{CAA}$ and VA injections was poor due to the relatively wide limits of agreement (standard deviations of CBV differences were 0.912 and $1.402 \mathrm{~mL} / 100 \mathrm{~g}$, respectively), with the magni-
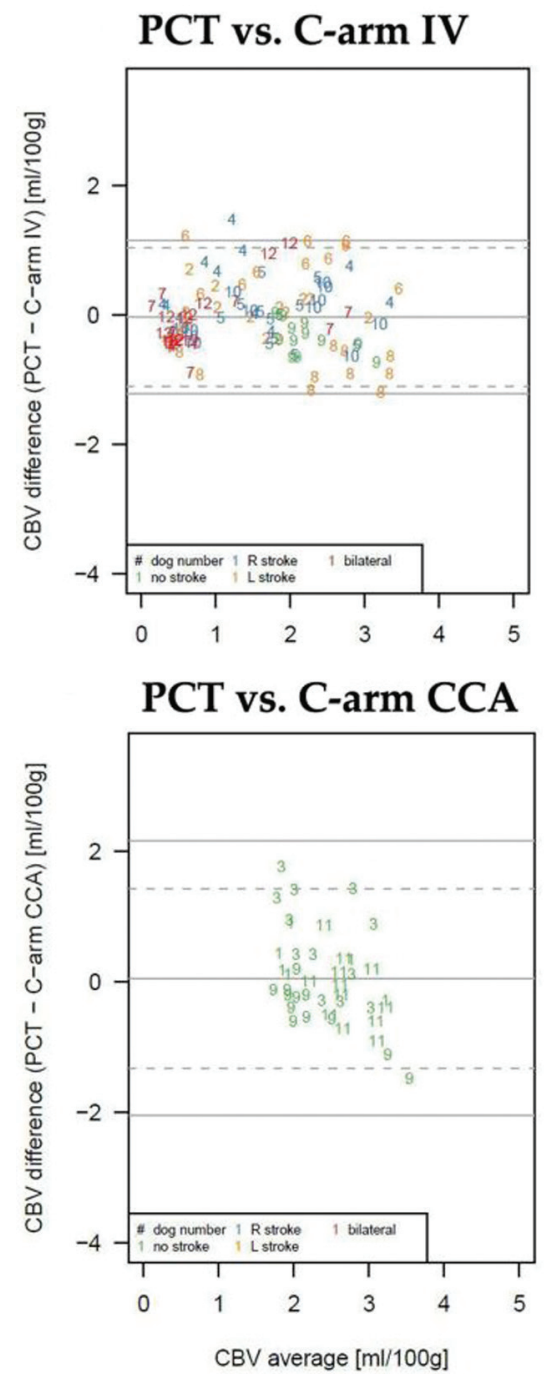

tude of the discrepancy being dependent on the injection site for the CBV being measured (CCA consistently underestimating CBV and VA injections consistently overestimating CBV) relative to PCT CBV (Fig 2).

\section{Discussion}

Our study demonstrates that the use of an AA injection of contrast medium for C-arm CT measurement of CBV allows for a substantial reduction of the contrast dose while also providing global CBV maps that are comparable with ones obtained using standard PCT. This then offers a potential alternative to the use of an IV injection of contrast when CBV measurements are made using C-arm CT. ${ }^{6,7}$ We have also shown that, in the canines with acute ischemia, regional measurement of CBV in conjunction with either a CCA or a VA injection of contrast was not a reliable method for providing CBV maps. In normal canines, however, the CCA and VA injection routes did provide regional CBV maps that were comparable with ones obtained with standard PCT.

What are the potential reasons for this difference? It seems that, in the animals with acute ischemia, the unreliability in using CCA or VA injection sites to measure regional CBV was

\section{PCT vs. C-arm AA}

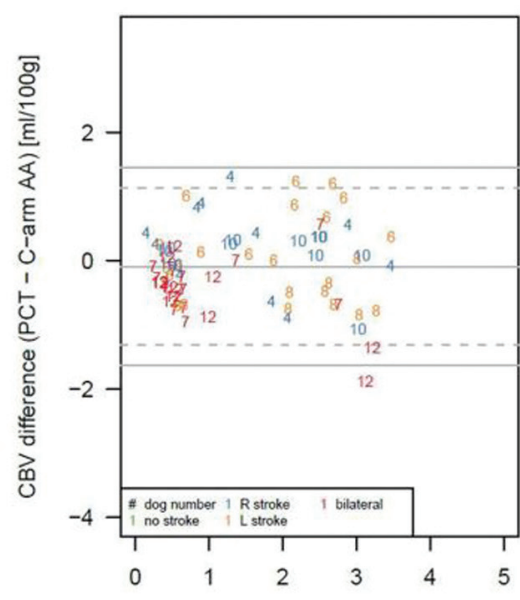

\section{PCT vs. C-arm VA}

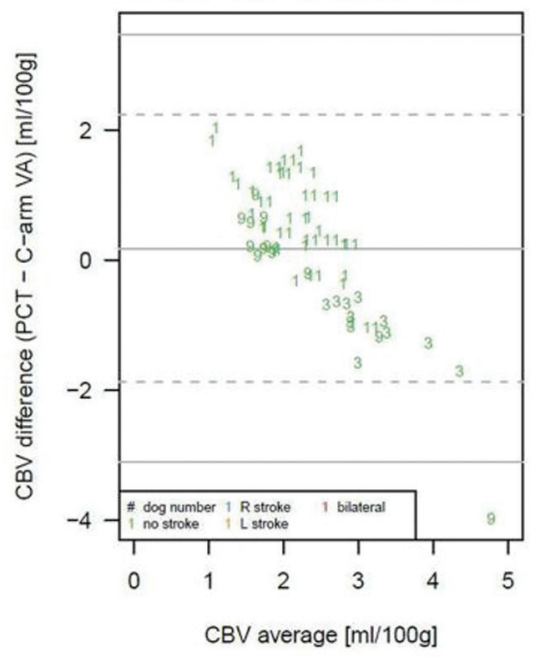

Fig 1. Bland-Altman plots. Modalities compared are shown at the top of each plot. 


\section{CBV vs. Hemisphere -Modality and Dog-}
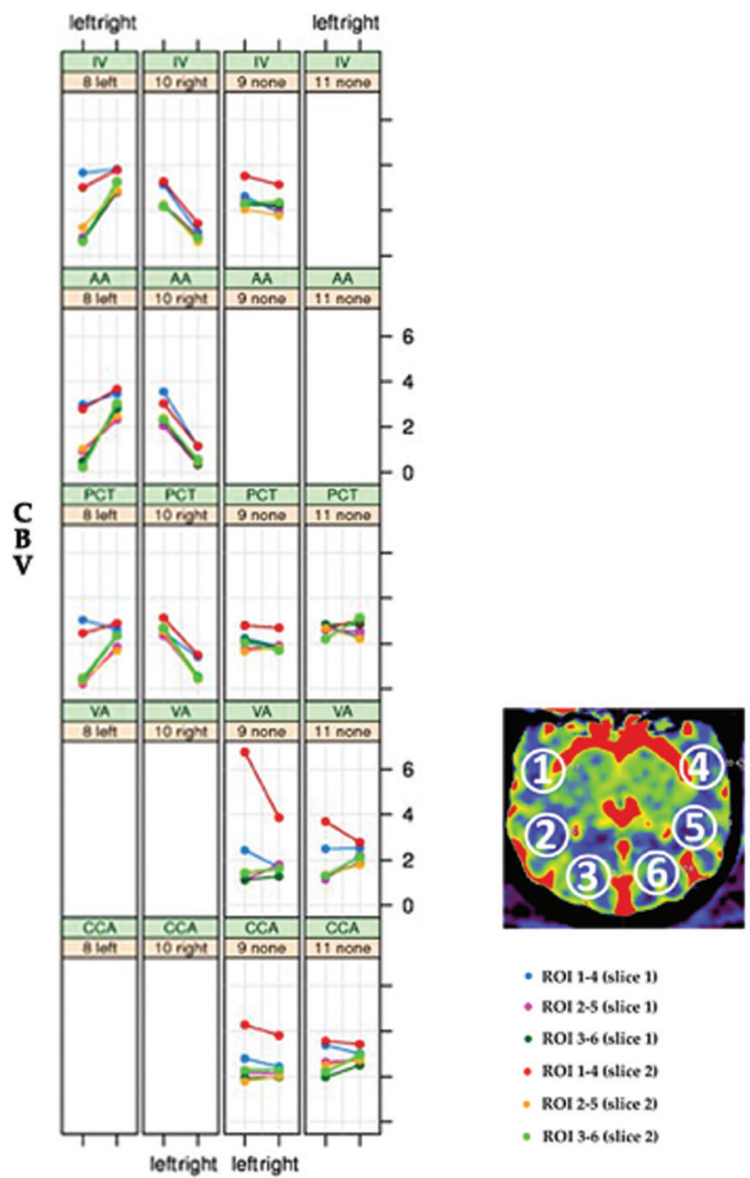

- ROI 1-4 (slice 1)

- ROI $2-5$ (slice 1$)$

- ROI 3.6 (slice 11

- ROI 1-4 (stice 2)

- ROI 2-5 (slice 2)

- RO1 3.6 (slice 2)

Fig 2. Scatterplots comparing injection sites and CBV values in normal and abnormal hemispheres in 2 normal animals and in 2 with ischemia. Analysis was not completed for the VA and CCA injections in the animals with a stroke. Each color depicts a different region of interest/section combination type (see key). The upper panel title contains the dog number and stroke laterality, and the lower panel title contains the type of CT injection. Line segments reflect pairing of ROls across hemispheres. The left column is from dog \#8, the second column from the left is from dog $\# 10$, the third column from the left is from dog $\# 9$, and the right column is from dog \#11. Because dog \#8 had a stroke, analysis for $\mathrm{C}$-arm VA injection was not performed in this dog.

due simply to an inability to deliver adequate amounts of contrast into the downstream tissue. Depending on the location of an obstruction, an injection into an artery ipsilateral to a stroke may fail to deliver adequate contrast, either because the injection site is proximal to an arterial obstruction, at a level where there is no collateral pathway around the blockage, or because there is either blockage of the collateral channels downstream from the injection site or no collateral channels exist. Injection into an artery contralateral to a stroke, or into one that has connections to the ischemic region, such as the VA in instances of ICA or MCA occlusions, may fail to deliver adequate contrast downstream from the injection site, either because of the absence of or blockage of collateral connections. It bears emphasis that there may also be situations when it appears that adequate contrast was not delivered to downstream tissue because the tissue of interest was receiving blood from some other arterial input; for example, consider a proximal left MCA occlusion in a situation where there is collateral

supply through both the anterior communicating artery and the posterior communicating artery; here, a "pseudo" perfusion abnormality might occur because of failure to fully opacify tissue from an injection into any single arterial input. Reliable measurement of $\mathrm{CVB}$ requires that all arterial inputs be taken into account; this is particularly relevant with C-arm CT acquisitions, where a steady state of tissue opacification throughout each acquisition is required if one is to obtain valid measurements. This incomplete filling or mixed filling by opacified and nonopacified blood from another arterial input probably accounts for the poor agreement of the absolute CBV values from maps made using a CCA or VA injection with ones made using PCT. Finally, slow delivery of contrast into an area of interest due to the length of collateral pathways might also produce a "pseudo" CBV abnormality if acquisition timing is not modified to account for this possibility.

Taking all of these factors into account, we believe that the most probable cause of our inability to successfully measure regional $\mathrm{CBV}$ using a CCA or a VA injection was due to the level of occlusions being at, or even proximal to, the circle of Willis, that is, the proximal MCA or distal ICA, and the lack of significant external carotid to internal carotid and leptomeningeal collaterals in the canine. ${ }^{19,20}$ Although the obvious differences between canine and human collaterals make it impossible to directly transfer our observations to humans, we are nonetheless somewhat doubtful about the ability of contrast injections made distal to the ascending aorta to reliably deliver evenly distributed contrast into tissue by way of collaterals, that is, to equally and constantly fill all inputs. However, because of the potential benefit of using selective IA injections for measurement of regional perfusion parameters, we stress that further evaluation of the technique in humans should be performed.

We elected to evaluate the possibility of using selective IA injections of contrast to measure CBV values in this study for several reasons. First, there was proved utility of using this parameter to help distinguish ischemic core from penumbra. ${ }^{21-24}$ Second, among the parameters CBV, CBF, and MTT, CBV has been shown to be the one with best agreement when measurements are made using a variety of postprocessing algorithms. ${ }^{24}$ Third, it has been demonstrated, in animals and in humans, that by using C-arm CT, measurements of CBV can be obtained that compare well with those made by using standard PCT. ${ }^{6-9}$ Finally, as we, and others, have previously noted, the potential to perform repeated assessments of perfusion parameters during an intervention seemingly would improve the ability of an operator to not only assess the effectiveness of treatment in an ongoing fashion - that is, whether a CBV abnormality is stable or whether it is becoming smaller-but also provide some insight into the benefit and risk of continuing an intervention, that is, whether the CBV abnormality is larger despite the intervention. The ability to perform these measurements on a regional basis, using injections of contrast through an existing catheter upstream from ischemic tissue, would seemingly further enhance the value of this capability.

\section{Conclusions}

Measurement of global CBV using C-arm CT in conjunction with either an IV or an AA injection was feasible in both normal animals and in ones with acute ischemia. Measurement of 
regional CBV with C-arm CT using either a CCA or VA injection, in normal canines, provided CBV maps that were qualitatively comparable with those obtained with PCT; however, the absolute CBV values from these maps were in poor agreement with those from the PCT measurements. Valid measurement of CBV using C-arm CT requires that all tissue in a target region be fully and equally opacified during any acquisition. Using selective CCA or VA injections, it was impossible for us to document if and when this had been achieved. CBV measurements using these routes of injection were therefore not reliable.

Disclosures: Charles Strother-RELATED: Grant: University of Wisconsin School of Medicine and Public Health, ${ }^{*}$ Comments: Study funded by Siemens Healthcare under an institutional Research Agreement Contract; UNRELATED: Consultancy: Siemens Healthcare AX, Comments: Unpaid advisor; Grants/Grants Pending: Siemens Healthcare AX; Travel/ Accommodations/Meeting Expenses Unrelated to Activities Listed: Siemens Healthcare AX, Comments: Travel to Advisors meetings is paid by vendor. Kevin Royalty—RELATED: Other: Siemens Medical Solutions, USA, Comments: I am a paid employee of Siemens Medical Solutions, USA, which collaborated with the University of Wisconsin on this research study. (*Money paid to institution)

\section{References}

1. Gomez C. Time is brain. J Stroke Cerebrovasc Dis 1993;3:1-2

2. Saver JL. Time is brain-quantified. Stroke 2006;37:263-66

3. Lees KR, Hankey GJ, Hacke W. Design of future acute-stroke treatment trials. Lancet Neurol 2003;2:54-61

4. Parsons MW, Pepper EM, Bateman GA, et al. Identification of the penumbra and infarct core on hyperacute noncontrast and perfusion CT. Neurology 2007;68:730-36

5. Murphy BD, Fox AJ, Lee DH, et al. White matter thresholds for ischemic penumbra and infarct core in patients with acute stroke: CT perfusion study. Radiology 2008;247:818-25

6. Ahmed AS, Zellerhoff M, Strother CM, et al. C-arm CT measurement of cerebral blood volume: an experimental study in canines. AJNR Am J Neuroradiol 2009;30:917-22

7. Bley T, Strother CM, Pulfer K, et al. C-arm CT measurement of cerebral blood volume in ischemic stroke: an experimental study in canines. AJNR Am J Neuroradiol $2010 ; 31: 536-40$

8. Struffert T, Deuerling-Zheng Y, Kloska S, et al. Flat detector CT in the evaluation of brain parenchyma, intracranial vasculature, and cerebral blood volume: a pilot study in patients with acute symptoms of cerebral ischemia. AJNR Am J Neuroradiol 2010;31:1462-69

9. Stuffert T, Deuerling-Zheng Y, Kloska S, et al. Cerebral blood volume imaging by flat detector computed tomography in comparision to conventional multislice perfusion CT. Eur Radiol 2011;21:882-89

10. Harris AD, Kosior BS, Chen HS, et al. Evolution of hyperacute stroke over 6 hours using serial MR perfusion and diffusion maps. J Magn Reson Imaging 2009;29:1262-70

11. Heidenreich JO, Hsu D, Wang G, et al. Magnetic resonance imaging results can affect therapy decisions in hyperacute stroke care. Acta Radiol 2008;49:550-57

12. Kosior JC, Ryder RC, Andersen LB, et al. MRI of ischemic stroke in canines: applications for monitoring intraarterial thrombolysis. J Magn Reson Imaging 2007;26:1421-28

13. Tudorica A, Fang Li H, Hospod F, et al. Cerebral blood volume measurements by rapid contrast infusion and T2*-weighted echo planar MRI. Magn Reson Med 2002;47:1145-57

14. Wintermark M, Flanders AE, Velthuis B, et al. Perfusion-CT assessment of infarct core and penumbra: receiver operating characteristic curve analysis in 130 patients suspected of acute hemispheric stroke. Stroke 2006;37:979-85

15. Schaefer PW, Roccatagliata L, Ledezma C, et al. First-pass quantitative CT perfusion identifies thresholds for salvageable penumbra in acute stroke patients treated with intra-arterial therapy. AJNR Am J Neuroradiol 2006;27: tients trea $20-25$

16. Bristow MS, Simon JE, Brown RA, et al. MR perfusion and diffusion in acute ischemic stroke: human gray and white matter have different thresholds for infarction. J Cereb Blood Flow Metab 2005;25:1280-87

17. Bland JM, Altman DG. Statistical methods for assessing agreement between two methods of clinical measurement. Lancet 1986;1:307-10

18. Bland JM, Altman DG. Agreement between methods of measurement with multiple observations per individual. J Biopharm Stat 2007;17:571-82

19. Clendenin MA, Conrad MC. Collateral vessel development after chronic bilateral common carotid artery occlusion in the dog. Am J Vet Res 1979;40: $1244-48$

20. Gillilan LA. Extra- and intra-cranial blood supply to brains of dog and cat. Am J Anat 1976;146:237-53

21. Koenig M, Kraus M, Theek C, et al. Quantitative assessment of the ischemic brain by means of perfusion-related parameters derived from perfusion CT. Stroke 2001;32:431-37

22. Hellier KD, Hampton JL, Guadagno JV, et al. Perfusion CT helps decision making for thrombolysis when there is no clear time of onset. J Neurol Neurosurg Psychiatry 2006;77:417-19

23. Lui YW, Tang ER, Allmendinger AM, et al. Evaluation of CT perfusion in the setting of cerebral ischemia: patterns and pitfalls. AJNR Am J Neuroradiol 2010;31:1552-63

24. Kudo K, Sasaki M, Yamada K, et al. Differences in CT perfusion maps generated by different commercial software: quantitative analysis by using identical source data of acute stroke patients. Radiology 2010;254:200-09 